\title{
Resolving lateral and vertical structures by ellipsometry using wavelength range scan
}

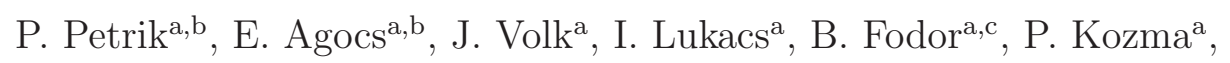

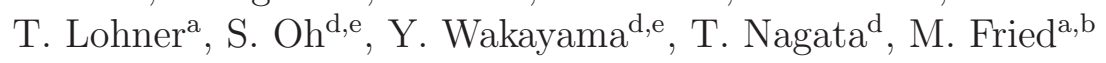 \\ ${ }^{a}$ Institute for Technical Physics and Materials Science (MFA), Research Center for \\ Natural Sciences, Konkoly Thege Rd. 29-33, 1121 Budapest, Hungary \\ ${ }^{b}$ Doctoral School of Molecular- and Nanotechnologies, Faculty of Information Technology, \\ University of Pannonia, Egyetem Rd. 10, Veszprem, H-8200, Hungary \\ ${ }^{c}$ Faculty of Science, University of Pécs, 7624 Pécs, Ifjuság Rd. 6, Hungary \\ ${ }^{d}$ International Center for Materials Nanoarchitectonics, National Institute for Materials \\ Science, 1-1 Namiki, Tsukuba 305-0044, Japan \\ ${ }^{e}$ Department of Chemistry and Biochemistry, Faculty of Engineering, Kyushu University, \\ 1-1 Namiki, Tsukuba 305-0044, Japan
}

\begin{abstract}
For most thin film structures, when changing the wavelength range to fit ellipsometric spectra, the values of the fitted parameters also change to a certain extent. The reason is that compared with the ellipsometric sensitivity many thin films are vertically non-uniform. In absorbing films with significant dispersion in the used wavelength range the penetration depth of probing light can show large variations depending on the wavelength. Consequently, the value of a fitted parameter for a certain wavelength range is a weighted sum of structural information over different depth ranges corresponding to the different wavelengths. When changing the wavelength range, the range of penetration depths can be adjusted. Next, the fitted values can be plotted as a function of the probed depth range calculated directly from the determined or tabulated extinction coefficients. We demonstrate the results on deposited polycrystalline thin films. The advantage of this approach over
\end{abstract}


the parameterization of structural properties as a function of depth is that the wavelength scan approach requires no parameterized depth distribution model for the vertical dependence of a layer property. The difference of the wavelength scan method and the vertical parameterization method is similar to the difference between the point-by-point and the parameterized dielectric function methods over the used wavelength range. The lateral structures also largely influence the ellipsometric response. One of the most characteristic effects is when the lateral feature sizes approach the wavelength of the probing light. In this case the effective medium method is not valid any more, scattering and depolarization occurs. When scanning the wavelength range, the limit wavelength of the onset of scattering can be found, and used for the determination of the corresponding critical lateral period length.

Keywords:

Spectroscopic Ellipsometry, depth profile, nanocrystal

\section{Introduction}

Thin films created by most frequently used techniques like deposition (sputtering, evaporation, ablation, etc.), etching (e.g. porous silicon) or ion implantation are usually non-uniform both laterally and vertically on the scale of ellipsometric sensitivity $[1,2,3,4]$ (sensitivity of a fit parameter is related here to the uncertainty, i.e. the $90 \%$ confidence limits of the fitted parameters). The vertical inhomogeneity is usually measured by dividing the surface region into numerous layers and fitting a characteristic parameter in each layer separately or as a defined function of depth $[5,6,2]$. In the first case we have a lot of fit parameters [7] (if a good depth resolution is 
aimed), whereas in the second case we have to assume a depth distribution function [6]. However, utilizing the fact that the penetration depth of light is a strong function of wavelength in semiconductors in the photon energy range around the critical points, a model independent "direct" depth scan can be performed.

The wavelength dependence can also be utilized for resolving lateral surface features if their period is comparable with the wavelength of illumination. The dielectric function of composite media can be calculated using the effective medium approximation (EMA), if the size of the distinct phases is significantly smaller than the wavelength of illuminating light. It has been shown by Egan and Aspnes that the EMA is considerably influenced for component sizes approaching the wavelength [8]. Recently, we showed that this effect can be used for the estimation of silicon nanosphere sizes comparable to the wavelength [4].

The aim of this article is to point out the potential of wavelength range scan for the resolution of both vertical and lateral features. The capabilities of vertical scan were demonstrated on a series of polycrystalline silicon layers with different thicknesses. In case of lateral features we show that the limit wavelength of EMA correlates with a characteristic lateral feature size in a resist pattern prepared by electron beam lithography.

\section{Experimental details}

Polycrystalline silicon [9] layers have been created using low pressure chemical vapor deposition on single-crystal Si wafers with $\approx 100 \mathrm{~nm}$ thermal oxide at a deposition temperature of $640^{\circ} \mathrm{C}$, pressure of $27 \mathrm{~Pa}$, and flow 


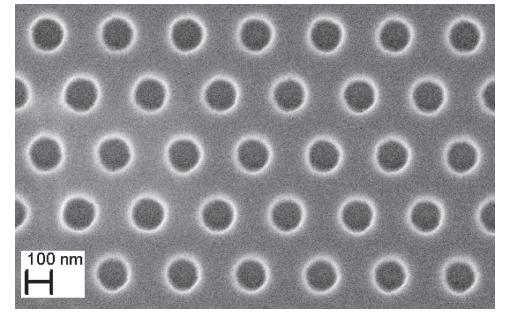

Figure 1: Micrograph of the sample with a hole size of nominally $120 \mathrm{~nm}$ and a period of $280 \mathrm{~nm}$ measured by scanning electron microscopy. The circular areas are exposed with e-beam and removed from the resist layer.

rate of $100 \mathrm{sccm}$. Polycrystalline silicon layers with the same deposition parameters but varied deposition times were created in the thickness range from 50 to $500 \mathrm{~nm}$.

For the studies of the lateral structure, crystalline silicon wafers were spin coated with $300 \mathrm{~nm}$ photoresist. Next, circular areas in a hexagonal order were removed from the resist using electron beam lithography (Fig. 1). The diameter of the circular part as well as the period has been varied in the range of 90-170 $\mathrm{nm}$ and 200-330 nm, respectively. Patterned areas as large as $0.3 \mathrm{~mm}$ by $0.6 \mathrm{~mm}$ were created so that the patterned area is suitable for the ellipsometric measurement with microspot.

We used a Woollam M-2000DI rotating compensator spectroscopic ellipsometer with a microspot of $0.3 \mathrm{~mm}$ beam diameter to measure the patterned areas. The ellipsometer is equipped with an $\mathrm{X}-\mathrm{Y}$ table that can be positioned with a precision of a few microns. Using ellipsometry [10] we measure the complex reflectance ratio $\rho=r_{p} / r_{s}=\tan \Psi e^{i \Delta}$, where $r_{p}$ and $r_{s}$ are the complex reflection coefficients of the light polarized parallel and perpendicular to the plane of incidence, respectively; $\Psi$ and $\Delta$ are the ellipsometric angles. 


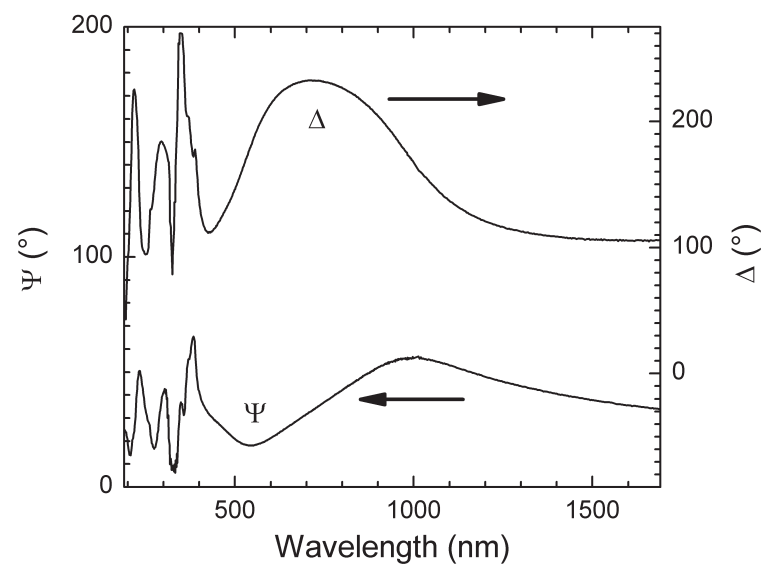

Figure 2: Measured ellipsometric angles on the sample created by e-beam lithography with a nominal hole size of $100 \mathrm{~nm}$. The angle of incidence was $60^{\circ}$. Note the irregular, distorted line shapes in the UV range.

Because the M-2000DI is a multichannel ellipsometer [11] that is capable of measuring $\Psi-\Delta$ spectra in the wavelength range of 193-1690 nm (Fig. 2) within a second with a precision of $0.05^{\circ}$ for both angles of $\Psi$ and $\Delta$, we could perform a high resolution (50 micron) mapping over an area of approximately $1 \mathrm{~mm}$ by $1 \mathrm{~mm}$ to locate the exact position of the patterned area within a few 10 minutes. To increase the sensitivity we measured at angles of incidence as small as possible to avoid that the measurement spot gets elongated too much and reaches the non-patterned area. The typical angle of incidence for the electron beam-patterned samples was $60^{\circ}$.

\section{Resolving vertical features}

The layer structure of the polycrystalline silicon wafers has been modeled using a single-crystalline $\mathrm{Si}$ (c-Si) substrate, an $\mathrm{SiO}_{2}$ layer created by 
thermal oxidation, a polycrystalline silicon layer with an effective medium composition of fine-grained polycrystalline silicon (nc-Si, [12]), c-Si and void (for density correction), as well as a surface roughness layer with $50 \%$ void and 50\% layer material. Fig. 3 shows the optical model and a typical fit on the thickest layer (tm25). The fitted model parameters for the different samples are compiled in Table 1 . In this study we focus on $f_{v}$, the volume fraction of void, which is a density correction of the polycrystalline silicon layer in the model. Note that it significantly changes with the layer thickness.

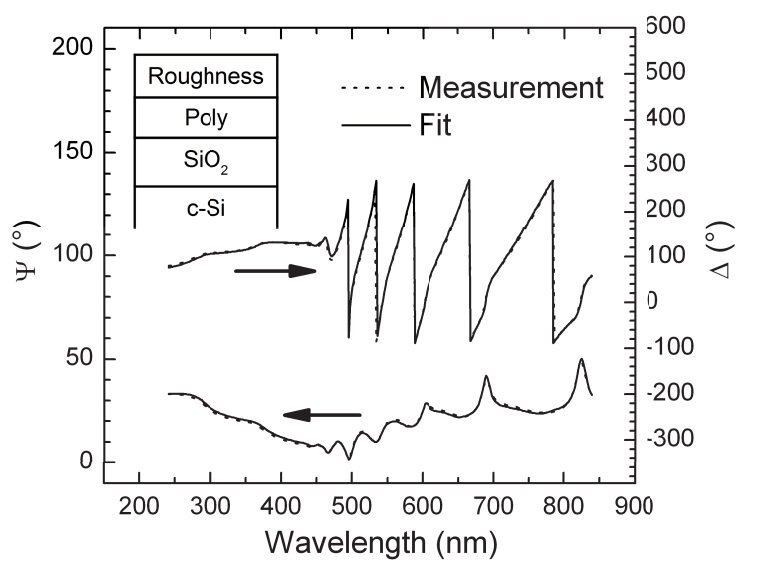

Figure 3: Measured and fitted $\Psi-\Delta$ spectra for the $\approx 500$-nm thick polycrystalline silicon sample. The angle of incidence is $75^{\circ}$. The inset shows the optical model. "Poly" denotes the polycrystalline silicon layer modeled by the effective medium composition of c-Si, nc-Si and void. The roughness layer is modeled by the effective medium composition of $50 \%$ void and $50 \%$ "Poly". In this study the wavelength range from 240 to $840 \mathrm{~nm}$ was used, because the nc-Si reference is only available for these wavelengths.

The variation of $f_{v}$ in depth can also be estimated using the fact that the penetration depth of illuminating light depends on the used wavelength range. The extinction coefficient $(k)$ of the polycrystalline silicon layer (which 
Table 1: Fitted parameters of polycrystalline silicon layers. Only the deposition time was varied to obtain changing layer thicknesses. The uncertainty of parameter fit for the volume fractions and layer thicknesses are below $1 \%$ and $1 \mathrm{~nm}$, respectively. $d_{b}$ and $d_{r}$ denote the thicknesses of the polycrystalline silicon layer and its surface roughness, respectively. $f_{c-S i}, f_{n c-S i}, f_{v, d}$, and $f_{v, d}^{(c)}$ denote the volume fractions of c-Si, nc-Si, void, and the corrected value of void, respectively. The fitted thickness of the buried oxide layer (not included in the table) is $\approx 120 \mathrm{~nm} . d_{b, c}$ is the depth which is equivalent with the penetration depth scale of Table 2 - see the method of calculation and the description in the text.

\begin{tabular}{|l|rrrrrrr|}
\hline Sample & $\begin{array}{r}d_{r} \\
(\mathrm{~nm})\end{array}$ & $\begin{array}{r}d_{b} \\
(\mathrm{~nm})\end{array}$ & $\begin{array}{r}d_{b, c} \\
(\mathrm{~nm})\end{array}$ & $\begin{array}{r}f_{c-S i} \\
\%\end{array}$ & $\begin{array}{r}f_{n c-S i} \\
\%\end{array}$ & $\begin{array}{r}f_{v, d} \\
\%\end{array}$ & $\begin{array}{r}f_{v, d}^{(c)} \\
\%\end{array}$ \\
\hline \hline $\operatorname{tm} 22$ & 3.0 & 57 & 436 & 49.7 & 48.2 & 2.1 & 2.1 \\
$\operatorname{tm} 23$ & 3.2 & 113 & 380 & 51.0 & 47.3 & 1.7 & 1.7 \\
$\operatorname{tm} 24$ & 4.0 & 276 & 217 & 38.8 & 56.9 & 6.0 & 4.2 \\
$\operatorname{tm} 25$ & 4.3 & 493 & 0 & 32.2 & 60.9 & 12.0 & 7.7 \\
\hline
\end{tabular}

is related to the absorption) has a characteristic feature around the $\mathrm{E}_{1}$ (360 $\mathrm{nm})$ and $\mathrm{E}_{2}(300 \mathrm{~nm})$ critical points, as shown in Fig. 4. The optical penetration depth $(\mathrm{OPD})$ in Fig. 4 was calculated using $\mathrm{OPD}=\lambda /(4 \pi k)$. From the wavelength of $\approx 370 \mathrm{~nm}$ to $\approx 550 \mathrm{~nm}$ the $\mathrm{OPD}$ increases from $\approx 20 \mathrm{~nm}$ to $\approx 500 \mathrm{~nm}$. Using wavelength ranges for the fit starting at $240 \mathrm{~nm}$ (lower limit of the nc-Si reference) to a wavelength varied in the above range, the penetration depth can be scanned throughout the thickness of the polycrystalline silicon layer. The range with the shortest wavelength which can still sense the boundary between the polycrystalline silicon and $\mathrm{SiO}_{2}$ layers can also be 
estimated by plotting the uncertainty for the thickness of the polycrystalline silicon layer (also shown in Fig. 4). This uncertainty rapidly increases for wavelengths that can not sense the boundary between the polycrystalline silicon and $\mathrm{SiO}_{2}$ layer. When the upper boundary of the wavelength range used for the fit is gradually decreased, the uncertainty of the fitted thickness of the polycrystalline silicon layer starts to increase at the wavelength of $\approx 490$ $\mathrm{nm}$, which is close to the wavelength of $550 \mathrm{~nm}$ (corresponding to OPD = $500 \mathrm{~nm})$ calculated from $k$.

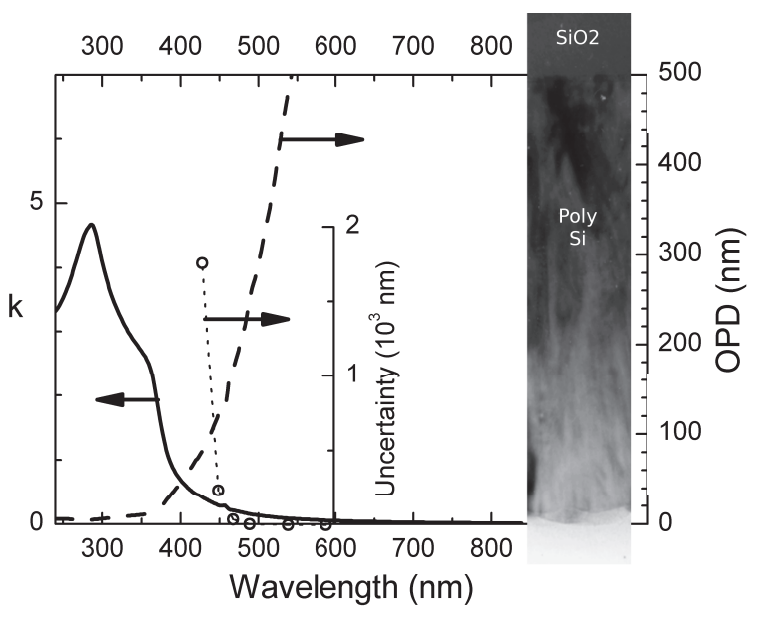

Figure 4: Extinction coefficient $(k)$ and optical penetration depth (OPD) measured for the polycrystalline silicon layer of sample tm25 as a function of the wavelength, as well as the uncertainty of the fitted thickness of the polycrystalline silicon layer as a function of the cut-off wavelength.

When calculating the volume fractions of the components as a function of the wavelength range (see Table 2), they can be assigned to certain depth ranges determined by the OPD. The behavior of the volume fraction of void $\left(f_{v}\right)$ is similar to that measured for polycrystalline silicon layers of differ- 
ent thicknesses (see Table 1). Note that in each wavelength range, $f_{v}$ is a weighted average over a depth range defined by OPD. The actual value at the given depth may be estimated using the following calculation. Let $f_{v, 1}$ denote the volume fraction of void in the first layer to a depth of $d_{1}$ from the surface in the narrowest wavelength range with the smallest penetration depth of light (see the first line of Table 2). Then the average value $f_{v, 2}$ of the second step to a depth of $d_{2}$ is

$$
f_{v, 2}=\left(f_{v, 1} d_{1}+f_{v, 1-2}\left(d_{2}-d_{1}\right)\right) / d_{2} .
$$

From equation 1 the actual value $f_{v, 1-2}$ in the depth range from $d_{1}$ to $d_{2}$ can be calculated as

$$
f_{v, 1-2}=\left(f_{v, 2} d_{2}-f_{v, 1} d_{1}\right) /\left(d_{2}-d_{1}\right) .
$$

Analogously, $f_{v, 2-3}$ in the depth range from $d_{2}$ to $d_{3}$ is

$$
f_{v, 2-3}=\left(f_{v, 3} d_{3}-f_{v, 2}\left(d_{3}-d_{2}\right)-f_{v, 1} d_{1}\right) /\left(d_{3}-d_{2}\right),
$$

and so on for the further ranges. In both Tables 1 and $2 f_{v, c}$ denotes the corrected values, which is plotted in Fig. 5 together with the raw values $\left(f_{v}\right)$. In Table 1, sample tm22 with the thinnest layer represents the initial stage of the deposition, so it represents the bottom part of the thickest layer (sample tm25) visible at longer wavelengths, as shown in Table 2. This means that in terms of OPD of Table 2 the 57-nm tm22 sample corresponds to the depth range of (493-57) to 493, sample tm23 (with thickness of $113 \mathrm{~nm}$ ) of Table 1 represents the depth region scanned in Table 2 between 493-113 and 493, and so on. Consequently, the depth ranges of the different depositions of Table 1 which correspond to the depth scan of Fig. 5 can be calculated as 
$d_{b, c}=d_{t m 25}-d_{b}$, where $d_{t m 25}$ is the thickness of the thickest layer. The values of $d_{b, c}$ are also compiled in Table 1 .

Table 2: Fitted parameters of sample tm25 with a polycrystalline silicon layer thickness of $493 \mathrm{~nm}$ using different wavelength ranges. $\lambda_{l}$ and $\lambda_{h}$ are the lowest and highest wavelengths, respectively. $k$ denotes the smallest value of the extinction coefficient in the used wavelength range, i.e. the value for the largest optical penetration depth (OPD) can be calculated by $O P D=\lambda /(4 \pi k) . f_{c-S i}, f_{n c-S i}, f_{v}$, and $f_{v, c}$ denote the volume fractions of c-Si, nc-Si, void, and the corrected value of void, respectively. The sensitivity for the volume fractions is below $1 \%$.

\begin{tabular}{|rr|rrrrrr|}
\hline $\begin{array}{r}\lambda_{l} \\
(\mathrm{~nm})\end{array}$ & $\begin{array}{r}\lambda_{h} \\
(\mathrm{~nm})\end{array}$ & $\mathrm{k}$ & $\begin{array}{r}\text { OPD } \\
(\mathrm{nm})\end{array}$ & $\begin{array}{r}f_{c-S i} \\
\%\end{array}$ & $\begin{array}{r}f_{n c-S i} \\
\%\end{array}$ & $\begin{array}{r}f_{v, w} \\
\%\end{array}$ & $\begin{array}{r}f_{v, w}^{(c)} \\
\%\end{array}$ \\
\hline \hline 240 & 410 & 0.600 & 54 & 15.3 & 74.4 & 10.3 & 10.3 \\
240 & 430 & 0.464 & 74 & 14.2 & 75.4 & 10.4 & 10.7 \\
240 & 450 & 0.338 & 106 & 14.0 & 75.6 & 10.4 & 10.6 \\
240 & 470 & 0.232 & 161 & 15.5 & 75.1 & 9.4 & 7.6 \\
240 & 490 & 0.155 & 252 & 24.7 & 68.0 & 7.3 & 2.4 \\
240 & 540 & 0.085 & 506 & 32.0 & 61.1 & 6.9 & 4.8 \\
240 & 590 & 0.052 & 903 & 33.4 & 59.8 & 6.8 & 5.3 \\
240 & 640 & 0.037 & 1377 & 33.7 & 59.5 & 6.8 & 6.8 \\
240 & 740 & 0.019 & 3099 & 33.4 & 59.8 & 6.8 & 6.8 \\
240 & 840 & 0.013 & 5142 & 32.2 & 60.9 & 6.9 & 7.1 \\
\hline
\end{tabular}

The agreement in the raw values between the results of wavelength scan (Table 2) and separate depositions (Table 1) are good, showing the same trends and similar values. The corrected values of the two cases $\left(d_{b, c}\right.$ of Table 1 and OPD of Table 2) don't agree well. A most probable explanation is that $f_{v, 1}, f_{v, 2}, \ldots$ are not average values over the depth range defined by the wavelength range, but they are strongly weighted, showing mainly the values close to the surface. Therefore, the most relevant comparison is probably 

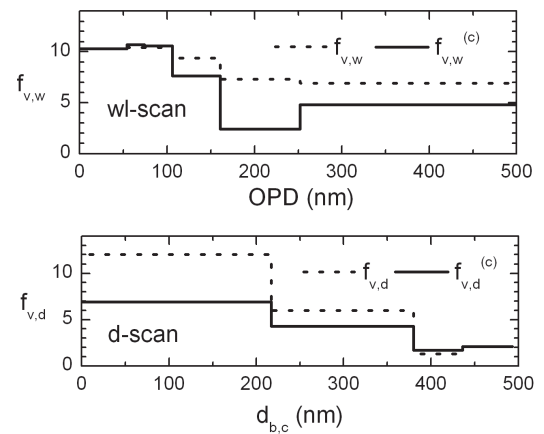

Figure 5: Raw $\left(f_{v, w}\right.$ of Table 2 and $f_{v, d}$ of Table 1$)$ and corrected $\left(f_{v, w}^{(c)}\right.$ of Table 2 and $f_{v, d}^{(c)}$ of Table 1) void profiles measured using wavelength scan on sample tm25 (top graph) and samples tm22-25 with different thicknesses (bottom graph). The top graph was plotted from Table 2, whereas the bottom graph from Table 1.

that of the raw curve of $f_{v, d}$ with the corrected profile of $f_{v, w}$ (Fig. 5).

Note that the most important feature of the wavelength range scan method is that it requires no assumption for the depth profile. The difference between the depth profiling using wavelength range scan and parameterized profiles is similar to the difference between the direct point-by-point inversion and the parameterized determination of the dielectric function. Using point-by-point inversion the dielectric function can be determined independently for each wavelength, which means that no assumed function is forced (fitted) to the spectrum. This allows the detection of detailed absorption features, which would not be possible when using smooth functions with a few parameters. Similarly, when a complicated depth distribution is assumed, the wavelength range scan can help to detect fine features. Furthermore, this method can also help to build a parameterized model based on the model-free profile 
determined by the wavelength range scan.

\section{Resolving lateral features}

To evaluate the patterned resist samples for lateral feature size characterizations, first we determined the refractive index of the resist layer on a non-patterned part of the sample using the Cauchy dispersion. The patterned layer was modeled using the isotropic Bruggeman EMA with two components: resist and voids. A possible anisotropy and depolarization is neglected in this study, although it may play a role, which is planned to be investigated in detail in a next investigation. We also took into account an interface layer with higher optical density (lower volume fraction of voids) which compensates in the cases the resist was not exposed through over the whole depth of the layer. In this model we fitted the layer thickness and the volume fraction of voids. The difference of the measured and fitted values are plotted in Fig. 6. The fit quality is good for the longer wavelengths, but there is an onset of the increase of difference towards the shorter wavelengths.

Although there might be a range of other useful algorithms to determine the boundary between the good-fit and bad-fit regions (wavelength $\lambda_{q}$ corresponding to the quasi-static limit of EMA [8]), an unambiguous method is the calculation of the second derivative of the mean square error (MSE) defined by

$$
M S E=\sqrt{\frac{1}{(2 n-p)} \sum_{j=1}^{n}\left\{\left(\frac{\Delta_{j}^{m}-\Delta_{j}^{c}}{\sigma_{j}^{\Delta}}\right)^{2}+\left(\frac{\Psi_{j}^{m}-\Psi_{j}^{c}}{\sigma_{j}^{\Psi}}\right)^{2}\right\}},
$$

in the wavelength range from $\lambda_{l}$ (lower wavelength limit) to $\lambda_{h}$ (highest wavelenth - in our case $1690 \mathrm{~nm}$ ) as a function of $\lambda_{l}$, where $n$ and $p$ are the 


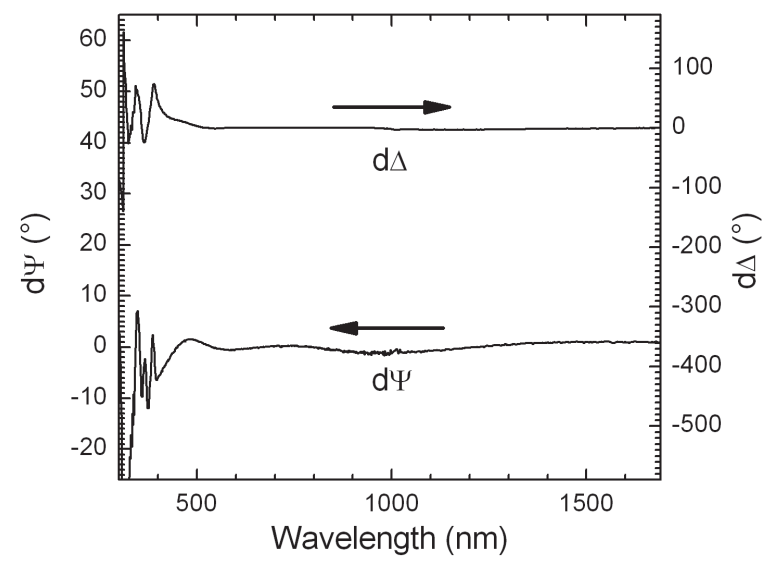

Figure 6: Difference of the fitted and measured $\Psi$ and $\Delta$ values in the whole wavelength range for the sample with a nominal hole size of $100 \mathrm{~nm}$. Note the well-defined onset of the increase of difference at $550 \mathrm{~nm}$.

number of independent measured data (total number of measured $\Psi$ values for all angles of incidence and wavelengths) and the number of fit parameters, respectively. $\Delta_{j}^{m}$ and $\Psi_{j}^{m}$ denote the measured, whereas $\Delta_{j}^{c}$ and $\Psi_{j}^{c}$ denote the calculated values. $\sigma$ stands for the standard deviations of the measured $\Psi$ and $\Delta$ ellipsometric angles. Using this approach well-defined peaks can be located corresponding to $\lambda_{q}$ in each case (Fig. 7), e.g. the peak in the second derivative corresponds to the wavelength at which MSE has the largest curvature.

Plotting $\lambda_{q}$ as a function of a characteristic feature size (the period $[P]$ minus the diameter $[D]$ of the circular holes) we could reveal a good correlation (Fig. 8). Using this correlation $P-D$ can be determined from $\lambda_{q}$ measured by a conventional ellipsometric setup. Note, that creating an area as large as $0.3 \mathrm{~mm}$ by $0.6 \mathrm{~mm}$ using e-beam lithography is a complex and time-consuming task. That is the reason for the relatively low number 


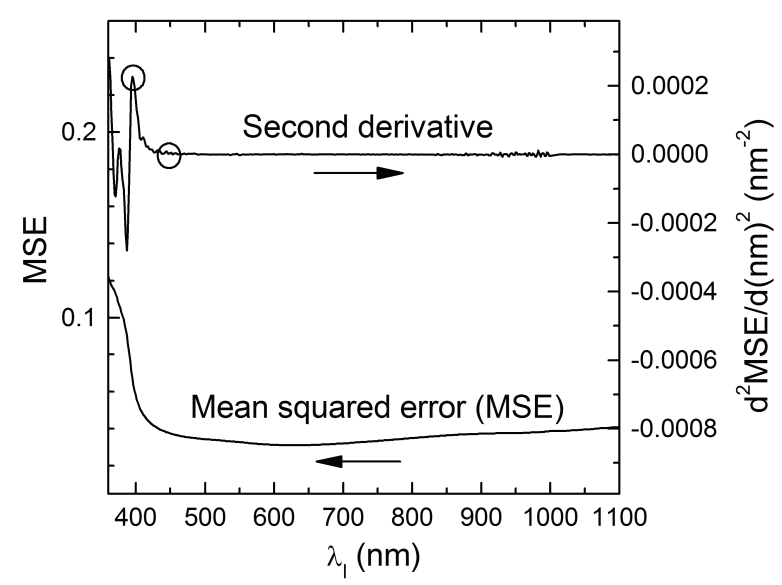

Figure 7: MSE (eq. 4) of the fit as a function of the smallest wavelength $\left(\lambda_{l}\right)$ of the fitted range (for the sample with a nominal hole diameter of $100 \mathrm{~nm}$ ). The vertical axis on the right-hand side shows the second derivative of MSE as a function of the wavelength.

of points in Fig. 8. However, to our opinion this 5 points are sufficient to indicate a good linearity.

\section{Conclusions}

It has been shown that the vertical and lateral inhomogeneity of thin films causes deviations in the fit results depending on the used wavelength range, especially in absorbing layers. This fact can be utilized when the fit is performed in systematically varied wavelength ranges taking into account the penetration depth of illuminating light. The method can be used as a depth scan, in which no assumption has to be made for the depth profile of the investigated component or property.

We have demonstrated that a characteristic lateral feature size can be measured using standard ellipsometric configurations with effective medium 


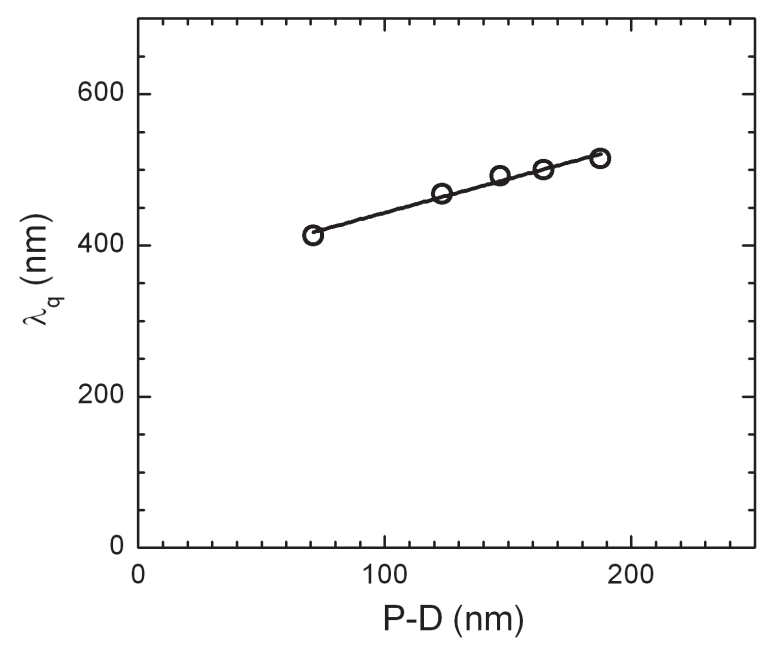

Figure 8: Wavelength of the quasi-static limit $\left(\lambda_{q}\right)$ as a function of the period $(P)$ minus the diameter $(D)$ of the circular holes in the resist. ( $P$ and $D$ were determined by SEM.)

models. Important features of this method are that it can be performed by most of the commercially available ellipsometers in a quick and robust way using simple effective medium models.

\section{Acknowledgements}

This work was supported by the National Development Agency grant TÁMOP-4.2.2/B-10/1-2010-0025, the OTKA grant Nr. K81842, Bilateral Joint Project involving Hungary and Japan established by the Japan Society for the Promotion of Science (JSPS), as well as by the János Bolyai Research Scholarship of the Hungarian Academy of Sciences. 


\section{References}

[1] P. Petrik, T. Lohner, M. Fried, L. P. Biró, N. Q. Khánh, J. Gyulai, W. Lehnert, C. Schneider, H. Ryssel, J. Appl. Phys. 87 (2000) 1734.

[2] P. Petrik, O. Polgár, M. Fried, T. Lohner, N. Khánh, J. Gyulai, J. Appl. Phys. 93 (2003) 1987.

[3] P. Petrik, M. Fried, E. Vazsonyi, P. Basa, T. Lohner, P. Kozma, Z. Makkai, J. Appl. Phys. 105 (2009) 024908.

[4] P. Kozma, B. Fodor, A. Deak, P. Petrik, Langmuir 26 (2010) 16122.

[5] J. Vanhellemont, P. Roussel, H. E. Maes, Nucl. Instr. Meth. 55 (1991) 183.

[6] M. Fried, T. Lohner, W. A. M. Aarnink, L. J. Hanekamp, A. van Silfhout, J. Appl. Phys. 71 (1992) 2835.

[7] P. Petrik, M. Fried, T. Lohner, O. Polgr, J. Gyulai, F. Cayrel, D. Alquier, J. Appl. Phys. 97 (2005) 1.

[8] W. G. Egan, D. E. Aspnes, Phys. Rev. B 26 (1982) 5313.

[9] P. Petrik, M. Fried, T. Lohner, R. Berger, L. P. Biró, C. Schneider, J. Gyulai, H. Ryssel, Thin Solid Films 313-314 (1998) 259.

[10] H. Fujiwara, Spectroscopic Ellipsometry: Principles and Applications, Wiley, New York, 2007.

[11] R. W. Collins, A. S. Ferlauto, in: E. G. Irene, H. G. Tomkins (Eds.), Handbook of ellipsometry, William Andrew, Norwich, NY, 2005. 
[12] G. E. Jellison, Jr., M. F. Chisholm, S. M. Gorbatkin, Appl. Phys. Lett. 62 (1993) 348. 